\title{
Mechanical Properties of Granular Soils: Triaxial versus Plane Strain Investigations
}

\author{
Andrzej Sawicki, Justyna Sławińska, Jacek Mierczyński, \\ Marcin Smyczyński
}

Institute of Hydro-Engineering, Polish Academy of Sciences, Kościerska 7, 80-328 Gdańsk, Poland, e-mails: stynaju@ibwpan.gda.pl,mier@ibwpan.gda.pl,m.smyczynski@ibwpan.gda.pl

(Received October 10, 2016; revised January 30, 2017)

\begin{abstract}
The paper compares the pre-failure behavior of granular soils investigated in the classical triaxial apparatus and in the true triaxial apparatus, under plane strain conditions. Both experiments are described within the framework of an incremental model of the pre-failure behavior of sands. The methods of tensor algebra are used to compare theoretical predictions with experimental results. The analysis presented deals with the pre-failure deformations of fully drained sand, as well as with its undrained behavior, including static liquefaction and the specific behavior of an initially dilative soil. Some key questions of soil mechanics are discussed, for instance, whether soil parameters determined from one configuration, such as triaxial conditions, can be applied in other cases.
\end{abstract}

Key words: mechanical properties of granular soils, triaxial investigations, true triaxial apparatus, liquefaction

\section{Introduction}

This paper deals with the most important issue in soil mechanics, namely with problems related to the experimental determination of the mechanical properties of granular soils. They are usually characterized by certain numbers, such as the angle of internal friction $\varphi$, the Young modulus $E$, and the Poisson ratio $v$. In saturated soils, an important coefficient is the soil skeleton permeability $k$. These numbers correspond to various aspects of soil behavior: $\varphi$ is related to the limit state of soils (their strength), $E$ and $v$ characterize their elastic response, and $k$ describes the filtration properties of the soil skeleton. The behavior of sands is very complex, so information provided by the above parameters is also limited. For this reason, many other theoretical models have been developed, each characterized by its own parameters, which have to be determined experimentally. As a result, there are hundreds of various coefficients 
characterizing sand behavior in different circumstances, and it is rather difficult to analyze even a fraction of them.

These various coefficients are usually determined in a triaxial apparatus, which is a standard piece of equipment in geotechnical laboratories. Some of these apparatuses are equipped with gauges for the measurement of deformations of soil samples and pore-water pressure, which provide valuable additional information about the deformability of soils and other aspects of their behavior, such as, liquefaction and related phenomena. Triaxial experiments deal with a specific axisymmetrical stress state, but their results are generalized to other configurations, and they are widely applied in geotechnical practice. Such procedures raise many questions that need to be answered. For example, whether the deformation characteristics of sand, determined in the triaxial apparatus, are sufficiently reliable to be applied in the modeling of plane strain problems? Note that most of geotechnical problems are formulated for plane strain conditions.

A chance for a more rational discussion about geotechnical coefficients appeared when more advanced experimental devices have become available, such as "true triaxial apparatuses" (TTA), in which plane strain conditions can be investigated. This is still a relatively new technology, and not much experience has been gained about its possibilities, except by a few research laboratories. Plane strain investigations are still not common practice in geotechnical engineering.

The aim of this paper is to investigate whether soil parameters determined with the classical triaxial apparatus (CTA), i.e. under axi-symmetrical conditions, can be applied in the analysis of experimental data obtained by TTA investigations for plane strain conditions. In order to compare these configurations, an incremental model of the pre-failure behavior of sand is applied. The model is calibrated under triaxial conditions and then validated for the plane strain configuration. This approach is consistent with the engineering mechanics methodology, as we use the methods of tensor algebra, including stress and strain invariants. Additionally, the results presented may be useful in interpretation of experimental results obtained from true triaxial apparatuses, which are not so common in geotechnical laboratories. Fig. 1 illustrates the idea of this paper.

\section{The Plane Strain Investigations}

According to Wanatowski and Chu (2007a), the importance of studying the stress-strain behavior of sand under plane strain conditions was noted as early as in the 1960s. Since then, several plane strain devices and even true triaxial cells have been developed, see Oda et al (1978), Tatsuoka et al (1986), Mokni and Desrues (1998). Some comparisons between triaxial and plane strain tests also have a long history. One of the basic discoveries is the following: "It was shown that the shear strength of granular soils under plane strain conditions is higher than that under axisymmetric stress conditions." Other investigations deal with the shear band formation, and various discrepancies 
Starting point: Incremental constitutive equations formulated for the triaxial configuration.

Calibration of these equations in a triaxial apparatus, see Sawicki, Świdziński (2010a, b).

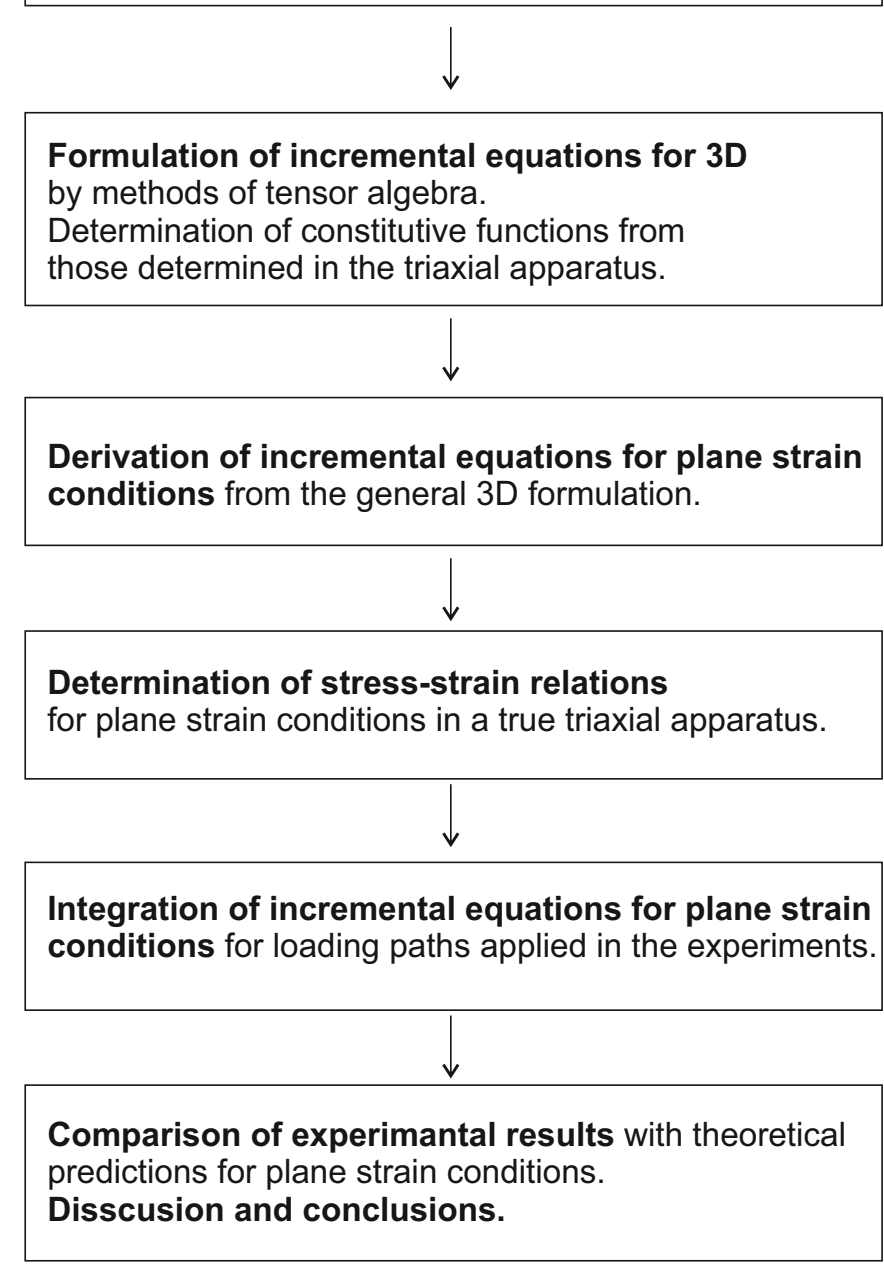

Fig. 1. Basic idea of this paper

between different laboratories have been observed. For references see Alshibli et al (2003), Finno et al (1997), Wanatowski and Chu (2007a).

More recent investigations also deal with comparisons between triaxial and plane strain tests. For example, Wanatowski and Chu (2007a) compare the drained behavior of sand in these two configurations and conclude that the angle of internal friction $\varphi$ is higher for the plane strain state than under axisymmetric conditions. They also conclude that the critical state lines (CSL) are different, and that those determined 
under axisymmetric conditions cannot be used for other configurations. In another paper, Wanatowski and Chu (2007c) study the problem of static liquefaction and conclude that the results obtained in the triaxial apparatus can be extended to plane strain conditions. A series of their papers is devoted to the problems of sand stability under plane strain conditions, see Chu and Wanatowski (2008), Chu and Wanatowski (2009), Wanatowski et al (2010), Wanatowski and Chu (2009), Wanatowski (2007). Another important problem studied by Wanatowski and Chu (2007b) concerns the $K_{0}$ coefficient under plane strain conditions.

\section{Incremental Model for the Triaxial Configuration}

The incremental model describing the pre-failure behavior of sand was originally proposed for the triaxial configuration, for details see Sawicki and Świdziński (2010a, b). This model was calibrated and then verified in a triaxial apparatus manufactured by GDS Instruments (UK). The apparatus is equipped with special gauges for the local measurement of vertical and horizontal strains and the pore water pressure $u$, see Menzies (1988). A soil sample prepared for experimental investigations is shown in Fig. 2.

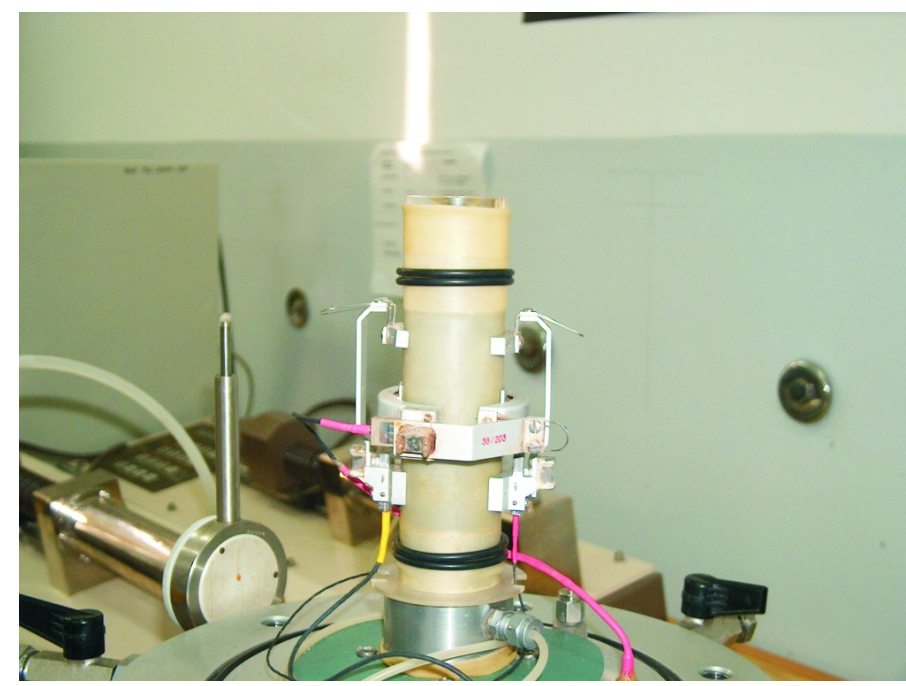

Fig. 2. A soil sample prepared for investigations in a GDS triaxial apparatus, photo W. Świdziński

The model describes the pre-failure deformations of drained sand, the behavior of saturated sand under undrained conditions, including such phenomena as the static liquefaction of initially contractive sand and the undrained behavior of initially dilative sand, as well as some other phenomena that characterize sand behavior, such as dilation and instabilities, see Sawicki and Świdziński (2010c), Sawicki (2011). 
The incremental model proposed is an alternative to other approaches, such as the elasto-plastic models of soils, which dominate in geotechnical engineering. The basic difference is that the total strains are considered, without distinguishing their elastic and plastic components. Therefore, it is unnecessary to introduce additional yield surfaces, which can hardly be shown experimentally. The only yield surface that remains unchanged is the Coulomb-Mohr failure condition. Another important difference is an alternative definition of loading/unloading phenomena. Instead of adopting the formalism of elasto-plasticity, we define two kinds of loading and unloading, related to the decomposition of the stress tensor into spherical and deviatoric parts. Subsequently, we introduce the spherical and deviatoric loading/unloading.

This section presents a summary of the incremental model for the triaxial configuration. For details, see the above-mentioned publications.

\subsection{Basic Equations}

The model is defined by the following incremental equations:

$$
\begin{aligned}
& d \varepsilon_{v}=\left(M+N_{p^{\prime}}\right) d p^{\prime}+N_{q} d q, \\
& d \varepsilon_{q}=\left(P+Q_{p^{\prime}}\right) d p^{\prime}+Q_{q} d q,
\end{aligned}
$$

where

$$
\begin{aligned}
& d \varepsilon_{v}=d \varepsilon_{1}+2 d \varepsilon_{3}-\text { increment of the volumetric strain, } \\
& d \varepsilon_{q}=2\left(d \varepsilon_{1}-d \varepsilon_{3}\right) / 3-\text { increment of the deviatoric strain, } \\
& d p^{\prime}=\left(d \sigma_{1}^{\prime}+2 d \sigma_{3}^{\prime}\right) / 3-\text { increment of the mean effective stress, } \\
& d q=d \sigma_{1}^{\prime}-d \sigma_{3}^{\prime}-\text { increment of the deviatoric stress, } \\
& M, N_{p^{\prime}}, N_{q}, P, Q_{p^{\prime}}, Q_{q}-\text { constitutive functions. }
\end{aligned}
$$

The soil mechanics sign convention is adopted, in which compression is positive.

The constitutive functions $M, N_{p^{\prime}}, N_{q}, P, Q_{p^{\prime}}, Q_{q}$ should be determined from experiments performed in the triaxial apparatus shown in Fig. 2. Their forms are different for spherical and deviatoric loading/unloading, which are as follows:

$$
\begin{array}{ll}
d p^{\prime}>0- & \text { spherical loading, } \\
d p^{\prime}<0- & \text { spherical unloading, } \\
d q>0- & \text { deviatoric loading, } \\
d q<0- & \text { deviatoric unloading. }
\end{array}
$$

\subsection{Experimental Determination of the Basic Stress-strain Characteristics}

Below, some experimental results for the "Skarpa" model sand are presented. Geotechnical characteristics of this sand are the following: mean particle size $D_{50}=0.42 \mathrm{~mm}$, 
uniformity coefficient $U=2.5$, minimum void ratio $e_{\min }=0.432$, maximum void ratio $e_{\max }=0.677, \varphi=34^{\circ}$ (loose sand) and $\varphi=41^{\circ}$ (dense sand), $\varphi$ was determined on the basis of investigations in a classical triaxial apparatus.

The model was calibrated for some simple effective stress paths shown in Fig. 3. They were selected to determine the reaction of sand to purely spherical or deviatoric loading and unloading.

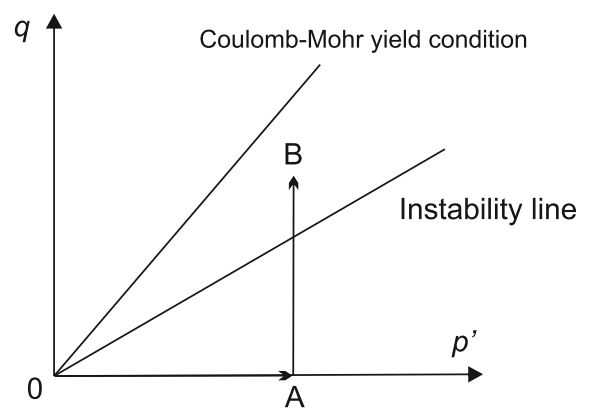

Fig. 3. Basic effective stress paths for the determination of functions $M, N, P$ and $Q$ in the classical triaxial apparatus

The path $0 \mathrm{~A}$ corresponds to purely spherical loading, and the path A0 defines spherical unloading. The deviatoric stress does not change along these paths, i.e. $d q=0$. Therefore, we can determine experimentally the strains $\varepsilon_{v}$ and $\varepsilon_{q}$ which develop along this particular stress path. Having found these strains, we can determine the functions $M$ and $P$ which appear in Eqs. (1) and (2):

$$
\begin{gathered}
M=\frac{d \varepsilon_{v}^{0 \mathrm{~A}}}{d p^{\prime}}, \\
P=\frac{d \varepsilon_{q}^{0 \mathrm{~A}}}{d p^{\prime}} .
\end{gathered}
$$

Another set of experiments dealt with pure shearing along the path $\mathrm{AB}$ at a constant mean effective stress $\left(d p^{\prime}=0\right)$. Those experiments made it possible to determine the functions $N_{p^{\prime}}, N_{q}, Q_{p^{\prime}}, Q_{q}$ in Eqs. (1) and (2):

$$
\begin{gathered}
N_{p^{\prime}}=\frac{\partial \varepsilon_{v}^{\mathrm{AB}}}{\partial p^{\prime}}, \\
N_{q}=\frac{\partial \varepsilon_{v}^{\mathrm{AB}}}{\partial q}, \\
Q_{p^{\prime}}=\frac{\partial \varepsilon_{q}^{\mathrm{AB}}}{\partial p^{\prime}},
\end{gathered}
$$




$$
Q_{q}=\frac{\partial \varepsilon_{q}^{\mathrm{AB}}}{\partial q} .
$$

Fig. 4 illustrates typical stress-strain characteristics during spherical loading (path $0 \mathrm{~A}$ in Fig. 3), which describe the functions $M$ and $P$. The character of the function $N_{q}$ (path AB in Fig. 3) depends on the initial (contractive or dilative) state of soil, see Fig 5.

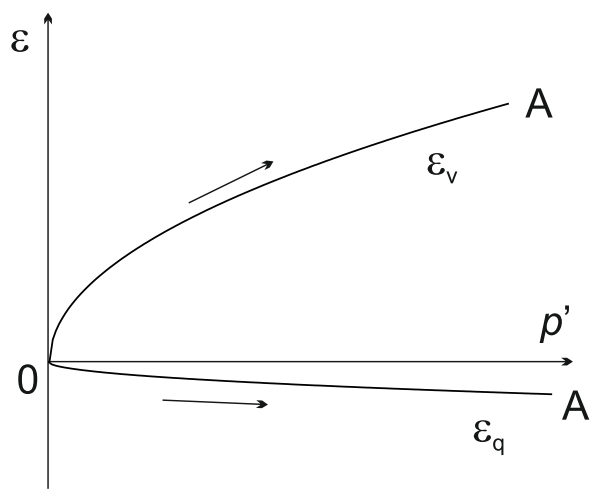

Fig. 4. The stress-strain characteristics of the "Skarpa" sand during spherical loading in the triaxial apparatus

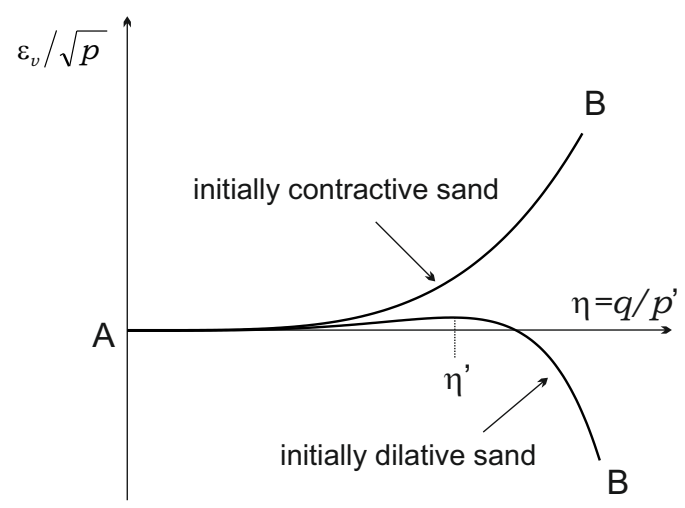

Fig. 5. Volumetric strains that develop during shearing at a constant mean effective stress (path $\mathrm{AB}$ in Fig. 3), initially dilative and contractive sands clearly differ in their behavior

The curves presented in Fig. 4 can be approximated by the following formulae:

$$
\varepsilon_{v}=A_{v} \sqrt{p^{\prime}} ; \quad \varepsilon_{q}=A_{q} \sqrt{p^{\prime}},
$$

where $A_{v}$ and $A_{q}$ are certain numbers (material parameters), see Sawicki and Świdziński (2010a). A similar procedure is applied for spherical unloading. During deviatoric 
loading ( $p^{\prime}=$ const, path $\mathrm{AB}$ in Fig. 3), it is convenient to present the experimental results in a new system of coordinates: $\varepsilon_{v} / \sqrt{p^{\prime}}$ and $\eta=q / p^{\prime}$, see Sawicki and Świdziński (2010a, b). Fig. 5 illustrates the character of stress-volumetric strain curves for initially contractive and dilative sands.

The experimental curves can be approximated by different functions. We have found that volumetric strains for initially contractive sand can be approximated by the following formula, see Sawicki and Świdziński (2010a):

$$
\frac{\varepsilon_{v}}{\sqrt{p^{\prime}}}=c_{1} \eta^{4}
$$

where $c_{1}$ is a coefficient. An approximation of the curve representing initially dilative sand (11) is more complex, as it exhibits two kinds of behavior. This sand initially compacts (up to $\eta^{\prime}$ ), and then it dilates, see Fig 5.

$$
\frac{\varepsilon_{v}}{\sqrt{p^{\prime}}}=a_{1} \eta^{3}\left(\eta-a_{2}\right) \exp \left(a_{3} \eta\right)
$$

where $a_{1}, a_{2}, a_{3}$ are coefficients.

The deviatoric strains that develop during pure shearing have a similar character sketched in Fig. 6. They asymptotically tend to infinity for $\eta^{\prime \prime}$, which corresponds to the limit state (steady state, when unconfined plastic flow takes place). The following relation approximates the experimental results:

$$
\frac{\varepsilon_{q}}{\sqrt{p^{\prime}}}=b_{1}\left(\exp \left(b_{2} \eta\right)-1\right)
$$

where $b_{1}, b_{2}$ are constants.

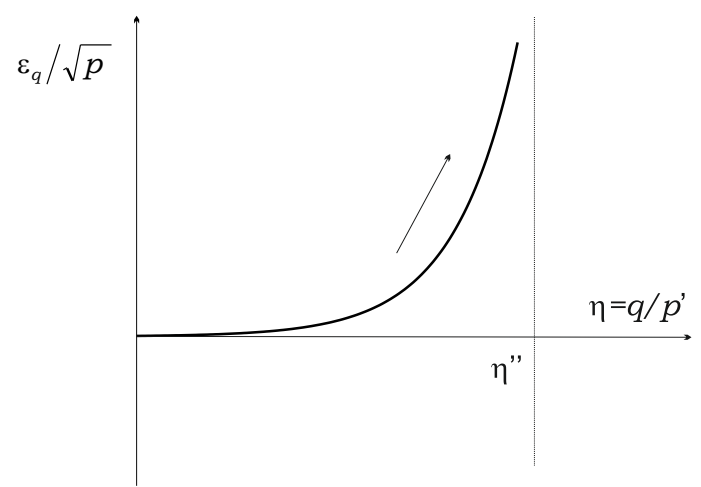

Fig. 6. The deviatoric strains that develop during pure shearing (path $A B$ in Fig. 3) in the triaxial apparatus

It is realized that some 10 coefficients are necessary to describe the stress-strain characteristics of sand before failure, which is a large number. The same number 
of coefficients is needed to describe unloading. However, at the beginning we have bare experimental data, well approximated by analytical formulae, which are useful in further research. Recall that the aim of this paper is to compare experimental results obtained from two different devices. A "common denominator" that makes such comparisons possible is the incremental model of the pre-failure behavior of soil. At present, the number of "constants" is irrelevant, as we are going to compare bare experimental data. The practical relevance of the results presented is another issue.

\subsection{Calibration of the Model}

The starting point for the calibration of the incremental equations are the analytical approximations of experimental data presented in the previous section. Recall that, in these equations, the mean effective stress is treated as a parameter. A simple differentiation of functions (9) leads to the following result:

$$
\begin{aligned}
& d \varepsilon_{v}=\frac{A_{v}}{2 \sqrt{p^{\prime}}} d p^{\prime}=M d p^{\prime}, \\
& d \varepsilon_{q}=\frac{A_{q}}{2 \sqrt{p^{\prime}}} d p^{\prime}=P d p^{\prime} .
\end{aligned}
$$

The other functions can be determined in a similar way. For example, for initially contractive sand, we obtain:

$$
\begin{gathered}
N_{q}=\frac{4 c_{1} \eta^{3}}{\sqrt{p^{\prime}}}, \quad Q_{q}=\frac{b_{1} b_{2} \exp \left(b_{2} \eta\right)}{\sqrt{p^{\prime}}}, \\
N_{p^{\prime}}=\frac{-3.5 c_{1} \eta^{4}}{\sqrt{p^{\prime}}}, \quad Q_{p^{\prime}}=\frac{b_{1}\left(\exp \left(b_{2} \eta\right)-2 b_{2} \eta \exp \left(b_{2} \eta\right)-1\right)}{2 \sqrt{p^{\prime}}} .
\end{gathered}
$$

\subsection{Useful System of Units and Values of Parameters}

We apply a practically useful system of units, in which the stress unit is $10^{5} \mathrm{~N} / \mathrm{m}^{2}=$ $100 \mathrm{kPa}$, and the strain unit is $10^{-3}$. For example, a stress of $500 \mathrm{kPa}$ will be presented in the form $5 \times 10^{5} \mathrm{~N} / \mathrm{m}^{2}=5 \times$ stress unit. A strain of $0.0003=0.3 \times 10^{-3}=0.3 \times$ strain unit. In our equations, we use only the values 5 and 0.3 . The values of particular parameters are determined for the above units. An advantage of this system of units is that we operate with numbers of a similar order of magnitude, which is useful in numerical calculations.

The values of coefficients depend mainly on the initial state of sand, which may be defined twofold. The classical division distinguishes between initially loose, medium dense, and dense sands, with some intermediate states. Such a classification may be found in every geotechnical textbook. The other classification distinguishes between initially contractive and dilative soils. Note that the same, for instance, medium dense, 
soil may display either contractive or dilative behavior, depending on the value of mean effective stress.

In order to illustrate the experimentally obtained values of parameters, let us consider $A_{v}$, see Eq. (9). It appears that for the "Skarpa" model sand, its values vary from 3.7 to 7.3 for loose sand, and from 2.2 to 4.6 for dense sand, see Sawicki and Świdziński (2010a). An attempt at a simplified statistical analysis of this problem is presented in Sawicki and Chybicki (2009). The parameter $A_{q}$ varies from -1.67 to -0.29 for loose sand, and from -1.02 to -0.12 for dense sand, see Sawicki and Świdziński (2010a). Note that for the same set of grains, it is impossible to form similar specimens for laboratory investigations. Therefore, such discrepancies are probably typical of soils.

Given the above data, consider Eq. (9) as an illustration of the system of units introduced. Assume $A_{v}=6$ and $p^{\prime}=200 \mathrm{kPa}=2 \times 10^{5} \mathrm{~N} / \mathrm{m}^{2}$. Eq. (9) leads to the following result: $\varepsilon_{v}=6 \sqrt{2}=8.485$, in the unit. Its physical value is 0.008485 .

Rough values of the other coefficients, obtained experimentally for the "Skarpa" sand, are presented below:

$$
\begin{aligned}
& a_{1}=-0.7, \quad a_{2}=-1.2, \quad a_{3}=2, \quad b_{1}=0.02, \quad b_{2}=6 \quad \text { (contractive sand), } \\
& b_{1}=4 \times 10^{-4}, \quad b_{2}=7 \text { (dilative sand). }
\end{aligned}
$$

It is realized that the number of these coefficients is large and difficult to determine in a standard geotechnical laboratory, as in the case of many other models of soils. Recall, however, that the incremental model of sand behavior is applied in order to compare experimental results obtained from two different apparatuses.

\section{3D Formulation of the Incremental Model}

\subsection{Basic Equations}

The following equations are a generalization of incremental constitutive equations (1) and (2) for a general 3D case, see Sawicki (2008):

$$
\begin{aligned}
& d \varepsilon_{v}=A d p^{\prime}+B \frac{\sqrt{3}}{2} \frac{d J_{2}}{\sqrt{J_{2}}}, \\
& d \boldsymbol{\varepsilon}^{d e v}=\mathbf{C} d p^{\prime}+D d \sigma^{d e v} .
\end{aligned}
$$

The stress $\sigma$ and strain $\varepsilon$ tensors are represented by their principal values, distinguished by subscripts ${ }_{1}, 2,3$, corresponding to the principal directions $x_{1}, x_{2}, x_{3}$, where $x_{1}$ denotes the vertical direction. Such notation corresponds to the structure of experimental apparatuses described in this paper.

Invariants in equations (17) and (18) are the following:

$$
\varepsilon_{v}=\varepsilon_{1}+\varepsilon_{2}+\varepsilon_{3}
$$




$$
\begin{gathered}
p^{\prime}=\frac{1}{3}\left(\sigma_{1}^{\prime}+\sigma_{2}^{\prime}+\sigma_{3}^{\prime}\right), \\
J_{2}=\frac{1}{3}\left(\sigma_{1}^{2}+\sigma_{2}^{2}+\sigma_{3}^{2}-\sigma_{1} \sigma_{2}-\sigma_{1} \sigma_{3}-\sigma_{2} \sigma_{3}\right) .
\end{gathered}
$$

Some new symbols are defined as follows:

$$
\begin{gathered}
\mathbf{C}=C \mathbf{S}^{d e v}, \\
\mathbf{S}=\left[\begin{array}{lll}
1 & 0 & 0 \\
0 & 0 & 0 \\
0 & 0 & 0
\end{array}\right]=\text { structural tensor, }
\end{gathered}
$$

$A, B, C, D=$ constitutive functions, depending on stress invariants and material parameters, which have to be determined experimentally. In the case considered, they will be determined from triaxial investigations, using the already determined functions $M, N, P$, and $Q$.

Note that the system of equations (17) and (18) consists of a scalar equation (17) and a tensor equation (18), equivalent to three scalar equations, for the case of principal directions. The structural tensor $\mathbf{S}$ is introduced to take into account the initial anisotropy effect, cf. the function $P$ in Eq. (2). In the case of an initially isotropic material, there is obviously $\mathbf{S}=\mathbf{0}$. Note that the initial anisotropy of sand is not taken into account in most of the existing models.

\subsection{Determination of Functions $A, B, C, D$}

First, let us consider Eqs. (1) and (17), which describe volumetric changes under triaxial and general 3D conditions, respectively. Substitution into Eq. (17) of the following functions:

$$
\sqrt{J_{2}}=\frac{q}{\sqrt{3}} \quad \text { and } \quad d J_{2}=\frac{2}{3} q d q,
$$

where these equations can be easily derived for the triaxial configuration, leads to the conclusion that Eqs. (1) and (17) are identical, which means that:

$$
A=M+N_{p^{\prime}}, \quad B=N_{q} .
$$

In order to determine the functions $C$ and $D$, let us consider Eq. (18) for the triaxial configuration $\left(\sigma_{2}=\sigma_{3}, \varepsilon_{2}=\varepsilon_{3}\right)$. For this case, the following relations hold:

$$
d \boldsymbol{\varepsilon}^{d e v}=\frac{1}{2}\left[\begin{array}{lll}
2 & & \\
& -1 & \\
& & -1
\end{array}\right] d \varepsilon_{q},
$$




$$
d \boldsymbol{\sigma}^{d e v}=\frac{1}{3}\left[\begin{array}{lll}
2 & & \\
& -1 & \\
& & -1
\end{array}\right] d q,
$$

and

$$
C=\frac{1}{3} C\left[\begin{array}{lll}
2 & & \\
& -1 & \\
& & -1
\end{array}\right] .
$$

Substitution of Eqs. (26)-(28) into Eq. (18) leads to a single scalar equation:

$$
d \varepsilon_{q}=\frac{2}{3} C d p^{\prime}+\frac{2}{3} D d q
$$

Comparison of Eqs. (2) and (29) gives:

$$
C=\frac{3}{2}\left(P+Q_{p^{\prime}}\right), D=\frac{3}{2} Q_{q} .
$$

\section{The Plane Strain State}

\subsection{Experimental Conditions}

The model is verified for plane strain conditions in a true triaxial apparatus manufactured by GDS Instruments, see Fig. 7. The chamber of the apparatus with a soil sample is shown in Fig. 8. Horizontal strain $\varepsilon_{3}$ is measured by local proximity transducers (see Fig. 7).

The configuration analyzed is the following: $\sigma_{1}$ is the vertical stress (controlled), $\sigma_{3}$ denotes the horizontal stress (controlled by the water pressure in the chamber). The corresponding strains, i.e. $\varepsilon_{1}$ and $\varepsilon_{3}$, are measured. The other principal stress $\sigma_{2}$ (reaction of the side plates) is measured. The role of the side plates is to prevent sample deformation in one direction $x_{2}$. There is $\varepsilon_{2}=0$.

Fig. 9 shows a soil sample, formed in a split mould, before installation in the chamber.

\subsection{Incremental Equations for the Plane Strain State}

The starting point are Eqs. (17) and (18), where $d \varepsilon_{2}=0$. The unknown increments are the following: $d \varepsilon_{1}, d \varepsilon_{3}$, and $d \sigma_{2}$. The system of governing equations consists of the scalar equation (17) and the tensor equation (18), which is equivalent to three scalar equations. It can be shown that one of these equations depends on the other two, and thus the system of equations (17) and (18) reduces to three scalar equations, so the problem is well defined (three unknowns and three equations). This system of equations can be written as follows: 


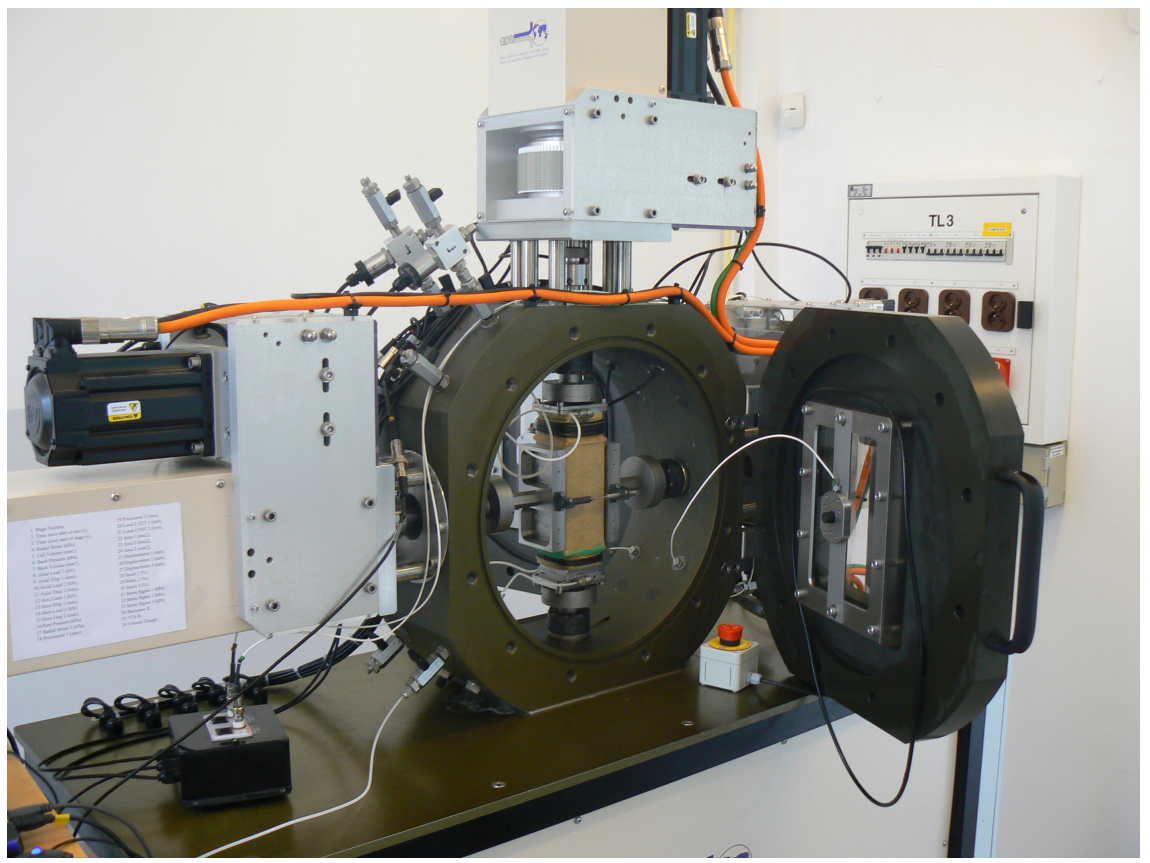

Fig. 7. A true triaxial apparatus manufactured by GDS Instruments

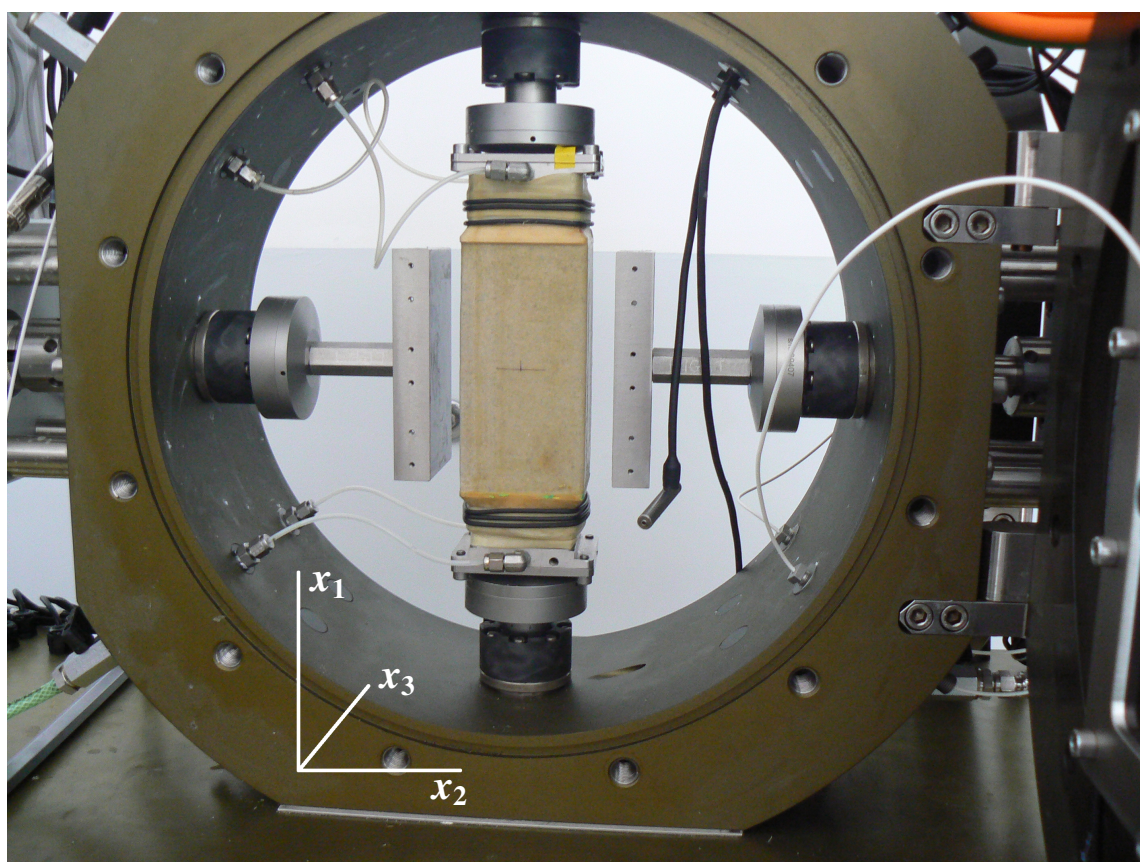

Fig. 8. The chamber of the true triaxial apparatus. The role of the side plates is to prevent soil deformations in $x_{2}$ direction 


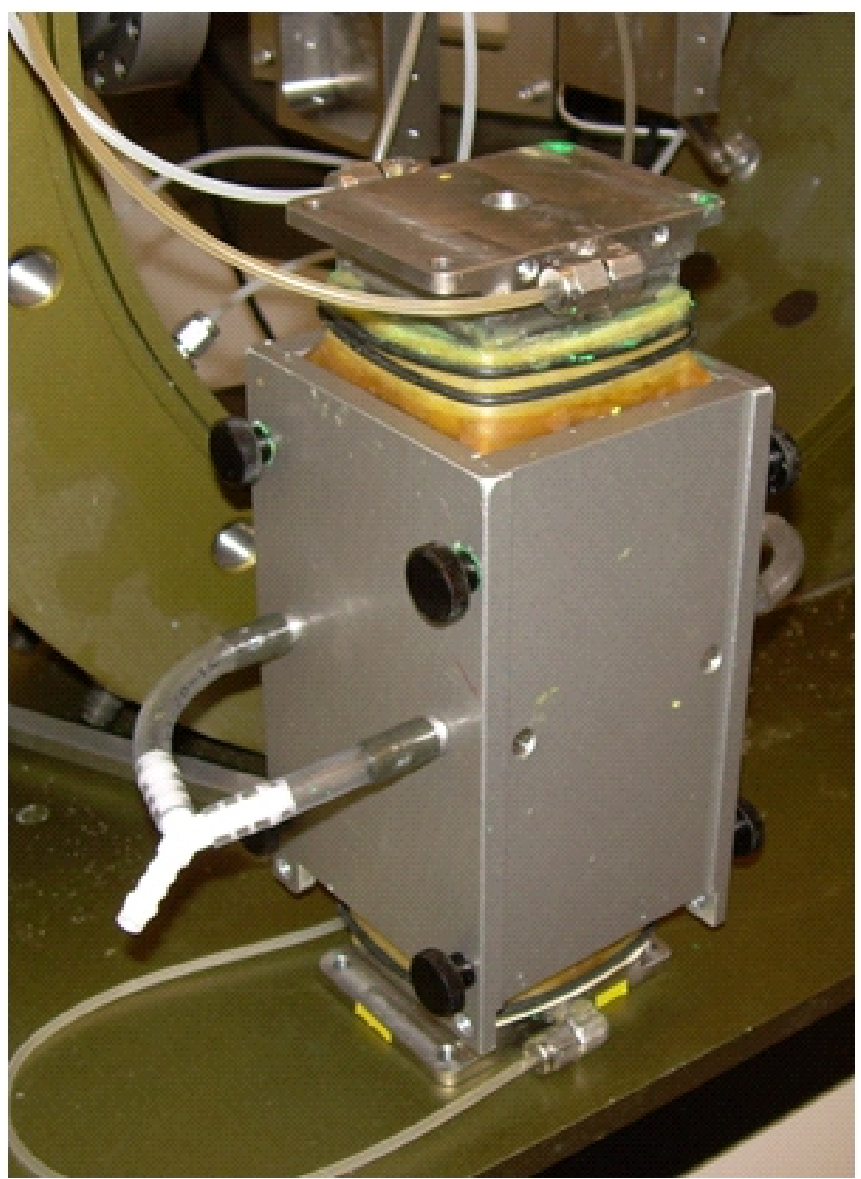

Fig. 9. A soil sample prepared in a split mould before installation in the chamber of the apparatus

$$
\begin{gathered}
2 d \varepsilon_{1}-d \varepsilon_{3}=A_{1} d \sigma_{1}+A_{2} d \sigma_{2}+A_{2} d \sigma_{3}, \\
-d \varepsilon_{1}-d \varepsilon_{3}=B_{1} d \sigma_{1}+B_{2} d \sigma_{2}+B_{1} d \sigma_{3}, \\
2 d \varepsilon_{3}-d \varepsilon_{1}=B_{1} d \sigma_{1}+B_{1} d \sigma_{2}+B_{2} d \sigma_{3}, \\
d \varepsilon_{1}+d \varepsilon_{3}=X d \sigma_{1}+Y d \sigma_{2}+Z d \sigma_{3},
\end{gathered}
$$

where:

$$
A_{1}=\frac{2}{3} C+2 D, A_{2}=\frac{2}{3} C-D, B_{1}=-\frac{1}{3} C-D, B_{2}=-\frac{1}{3} C+2 D,
$$




$$
\begin{aligned}
X=\frac{1}{3} A+\frac{\sqrt{3}}{3} a B, Y & =\frac{1}{3} A+\frac{\sqrt{3}}{2} b B, Z=\frac{1}{3} A+\frac{\sqrt{3}}{2} c B, \\
a & =\frac{2 \sigma_{1}-\sigma_{2}-\sigma_{3}}{\sqrt{3 J_{2}}}, \\
b & =\frac{2 \sigma_{2}-\sigma_{1}-\sigma_{3}}{\sqrt{3 J_{2}}}, \\
c & =\frac{2 \sigma_{3}-\sigma_{1}-\sigma_{2}}{\sqrt{3 J_{2}}} .
\end{aligned}
$$

If we add Eqs. (31)-(33) together, the identity $0=0$ will be obtained, which means that one of these equations depends on the other two.

\section{Pre-Failure Deformations under Plane Strain Conditions}

The basic question is how well the system of equations (31)-(34) predicts the pre-failure deformations of sand. Recall that these equations were derived from general 3D relations, which were calibrated in the triaxial apparatus. Eqs. (31)-(34) were integrated for given stress paths, and then these predictions were compared with the experimental results corresponding to the same loading paths. The results of a single experiment will be described in detail to illustrate the methodology proposed. These results correspond to dry, initially very loose "Skarpa" sand $\left(I_{D}=0.08\right)$ tested at a constant rate of vertical deformation.

Initially, the sample was subjected to isotropic compression up to $p=p^{\prime}=3$, expressed in the stress unit $10^{5} \mathrm{~N} / \mathrm{m}^{2}$. Then, the water pressure in the chamber was kept constant, i.e. $\sigma_{3}=3=$ const, and the vertical stress $\sigma_{1}$ was increased until the sample failed. The other principal stress $\sigma_{2}$ (reaction of the side plates) as well as the principal strains $\varepsilon_{1}$ and $\varepsilon_{3}$ were recorded. Recall that $\varepsilon_{2}=0$.

\subsection{Experimental Results}

Fig. 10 illustrates changes in $\sigma_{2}$ as a function of $\sigma_{1}$, when $\sigma_{3}=3=$ const. Note that in this kind of tests performed in TTA (plane strain conditions) we do not control the stress path, which we did in CTA. The intermediate principal stress $\sigma_{2}$ is measured. Fig. 11 shows the strains $\varepsilon_{1}$ and $\varepsilon_{3}$ measured during the experiment. Fig. 12 illustrates volumetric and deviatoric strains expressed by the second invariant of the strain deviator $\sqrt{K_{2}}$, where in $3 \mathrm{D}$ conditions:

$$
K_{2}=\frac{1}{3}\left(\varepsilon_{1}^{2}+\varepsilon_{2}^{2}+\varepsilon_{3}^{2}-\varepsilon_{1} \varepsilon_{2}-\varepsilon_{1} \varepsilon_{3}-\varepsilon_{2} \varepsilon_{3}\right) .
$$




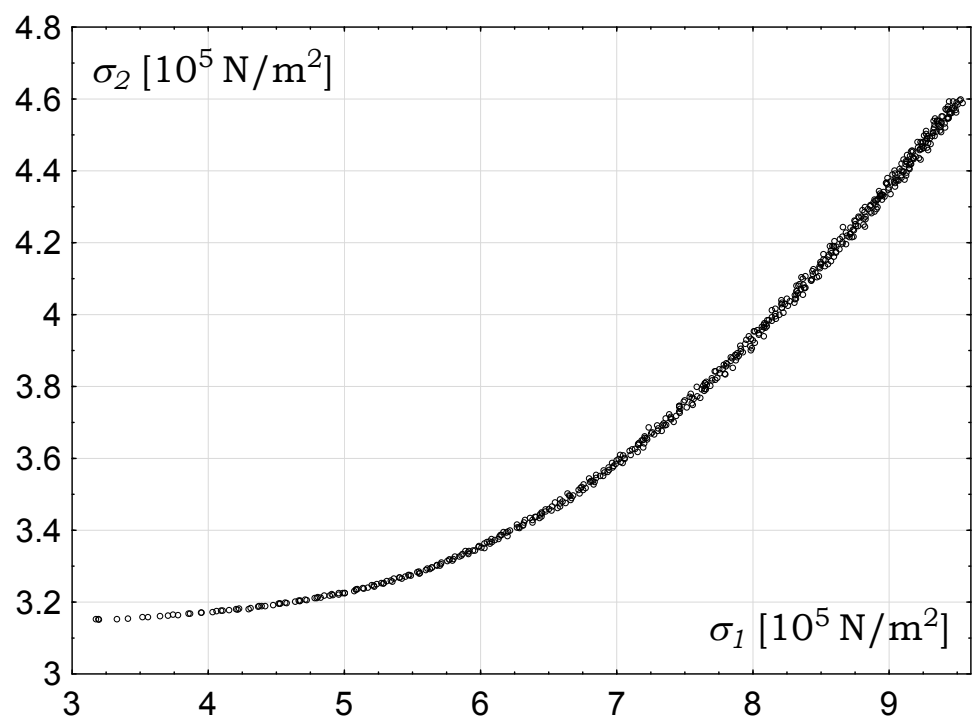

Fig. 10. The stress path $\sigma_{1}, \sigma_{2}$ during the experiment described. The initial stress state: $\sigma_{1}=\sigma_{2}=\sigma_{3}=3$

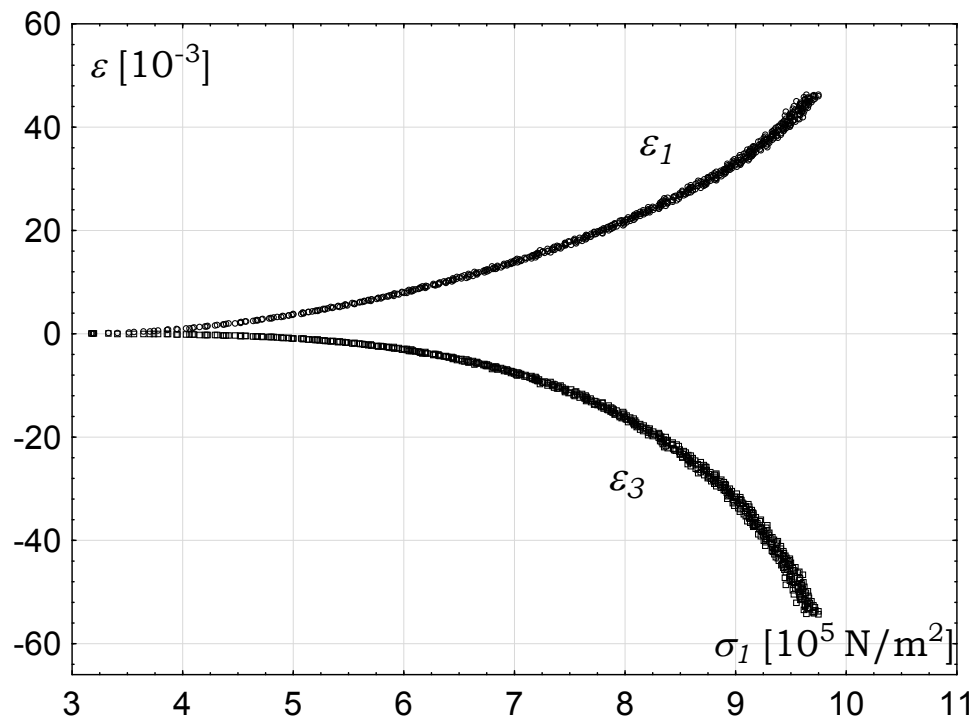

Fig. 11. Strains $\varepsilon_{1}$ and $\varepsilon_{3}$, measured during the experiment, as functions of the vertical stress $\sigma_{1}$

Recall that for the plane strain configuration analyzed in this paper, we have

$$
K_{2}=\frac{1}{3}\left(\varepsilon_{1}^{2}+\varepsilon_{3}^{2}-\varepsilon_{1} \varepsilon_{3}\right)
$$




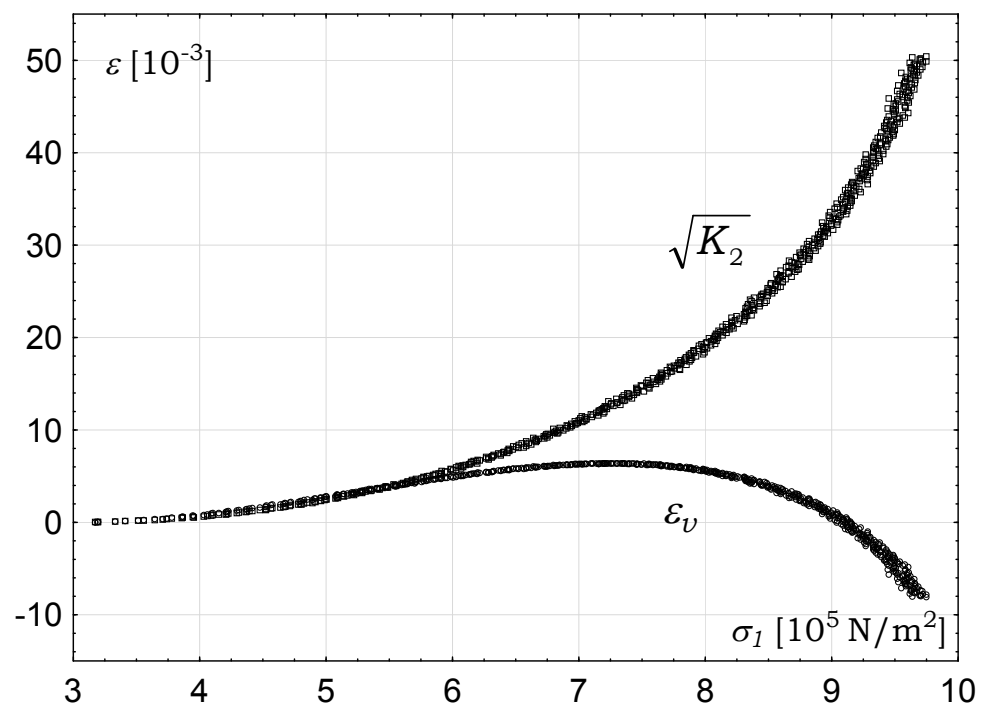

Fig. 12. Volumetric and deviatoric strains versus the vertical stress $\sigma_{1}$

Fig. 12 shows that the sand behavior is dilative, cf. Fig. 5. The maximum compressive volumetric strain corresponds to $\xi=\xi^{\prime} \approx 0.5$, where $\xi$ is a non-dimensional variable:

$$
\xi=\frac{\sqrt{J_{2}}}{p^{\prime}} .
$$

Recall that there is the following relation between the variable $\xi$ and the non-dimensional stress variable $\eta$ introduced in the analysis of triaxial investigations:

$$
\eta=\sqrt{3} \xi
$$

According to Eq. (38), $\eta^{\prime}=\sqrt{3} \xi^{\prime}=0.87$, which is very close to triaxial experimental data, see Sawicki and Świdziński (2010a, b). This number characterizes the slope of the instability line, so the agreement between triaxial and plane strain investigations is quite good.

\subsection{Theoretical Predictions}

The second part of this work deals with the integration of Eqs. (31)-(34) to determine the unknown functions $\varepsilon_{1}, \varepsilon_{3}$, and $\sigma_{2}$. Recall that one of these equations depends on the others, so in fact we have a system of 3 equations for 3 unknowns. The results of computations depend on the values of parameters characterizing the sand, and determined from triaxial investigations. Our set of data is limited, but sufficient to establish the order of magnitude of these parameters. Parameters characterizing particular 
samples may differ, as it is impossible to build the same samples from the same set of grains. Discrepancies between the values of soil parameters for apparently similar samples are quite large, as previously shown. Therefore, we have decided to perform a parametric study, using values of soil parameters close to those previously reported. Figs. 13-15 illustrate such an attempt, to fit theoretical predictions into experimental data, for the following data:

$$
A_{v}=6, \quad b_{1}=0.09, \quad b_{2}=5.2, \quad A_{q}=-0.5, \quad c_{1}=3 .
$$

These values are very close to parameters determined for loose "Skarpa" sand, see Sawicki and Świdziński (2010a).

In TTA, the stresses $\sigma_{1}, \sigma_{3}$ are controlled, but $\sigma_{2}$ is only measured. Equations (31)-(34) provide information about the behavior of $\sigma_{2}$. Fig. 13 presents the stress path: experimental results versus theoretical prediction expressed in terms of stress invariants (which are functions of $\sigma_{1}, \sigma_{2}, \sigma_{3}$ ).

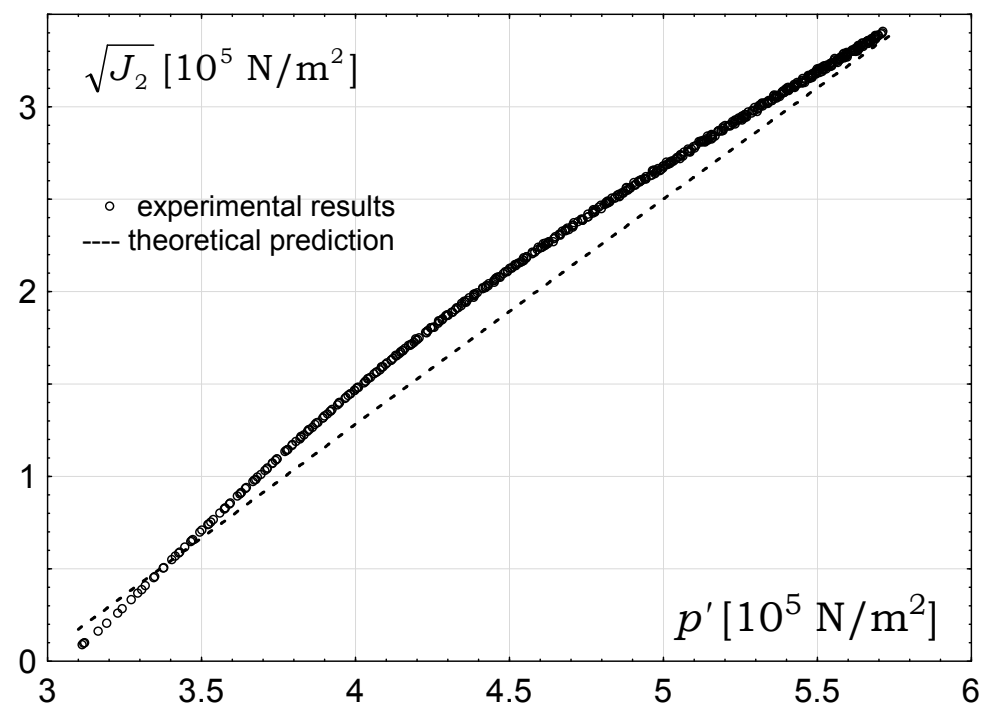

Fig. 13. The stress path in the space of invariants, experimental results versus theoretical prediction

Fig. 14 illustrates the vertical and horizontal strains $\varepsilon_{1}, \varepsilon_{3}$ : experimental results versus theoretical prediction (equations (31)-(34)). For example, Fig. 15 shows experimental results and prediction in the space of invariants, where $\sqrt{K_{2}}$ corresponds to the deviatoric strain.

\section{Static Liquefaction}

Static liquefaction has attracted the attention of many researchers for decades, see Jefferies and Been (2006), Lade and Yamamuro (1999), Madabhushi et al (2010), Sumer 


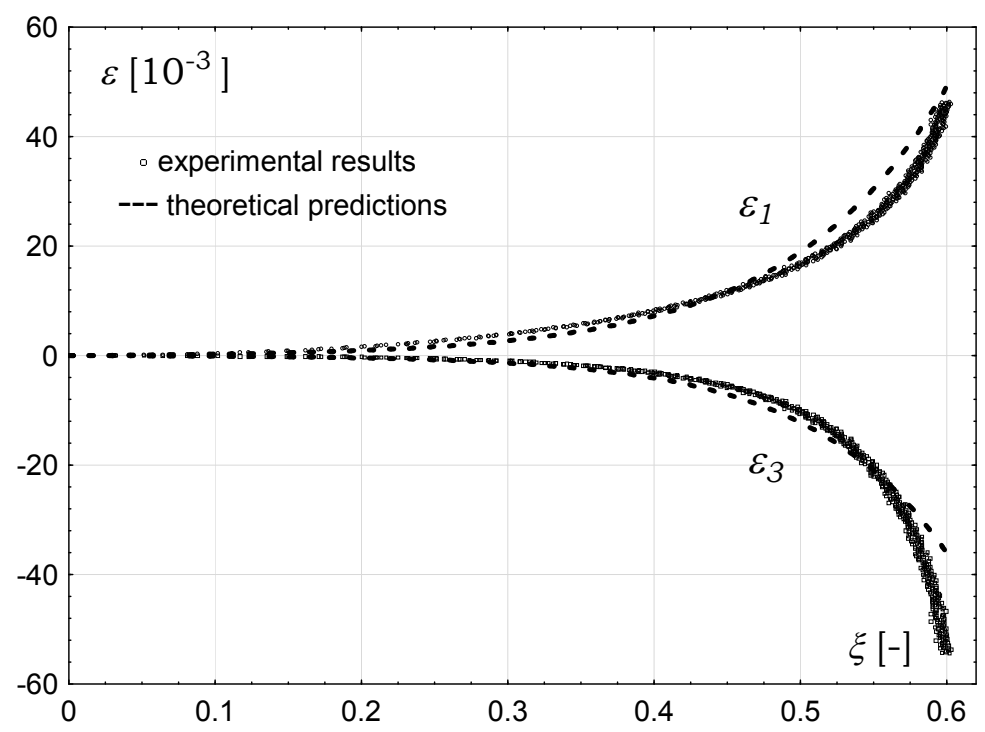

Fig. 14. Vertical and horizontal strains: experiment versus theoretical prediction

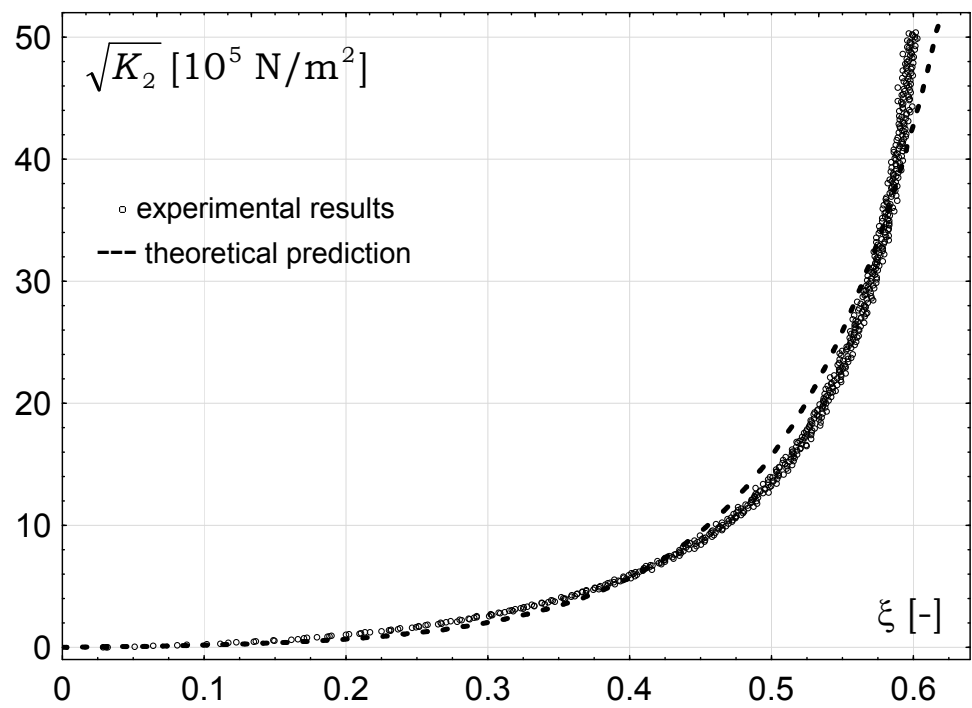

Fig. 15. Deviatoric strain: experiment versus theoretical prediction

(2014). This problem was also a subject of experimental and theoretical investigations by Sawicki and Świdziński (2010a, b), who used the incremental model of saturated soil tested in the triaxial apparatus. Recall that for the triaxial configuration, a good agreement was achieved between experimental results and theoretical predictions. In 


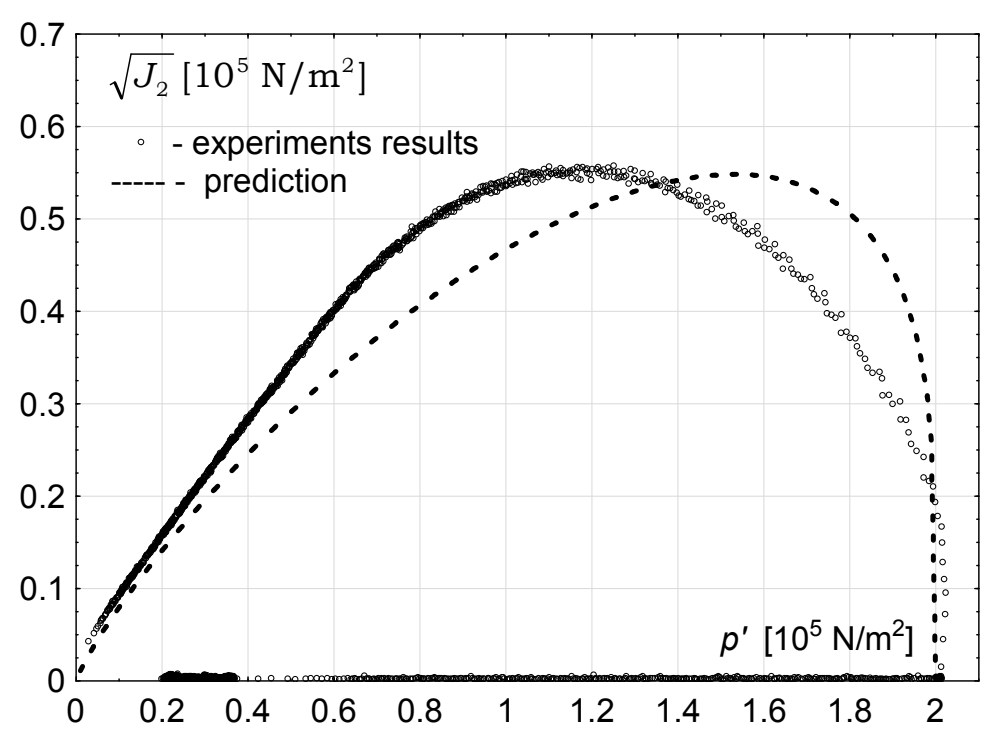

Fig. 16. Static liquefaction under plane strain conditions, experiment versus theoretical prediction

this section, the experimental results of plane strain investigations performed in TTA will be compared with theoretical predictions.

The experiments were performed on a fully saturated "Skarpa" model sand with the initial density $I_{D}=0.15$. First, the sample was subjected to isotropic pressure, up to $p^{\prime}=2$, expressed in the stress unit $10^{5} \mathrm{~N} / \mathrm{m}^{2}$. Then, the valves were closed to achieve undrained conditions. Subsequently, the vertical stress $\sigma_{1}$ was increased, and the excess pore water pressure was measured. The effective stress path followed during a single experiment is shown in Fig. 16. It has a well-known shape, as the deviatoric stress first increases to its maximum value $\left(\sqrt{J_{2}}\right)_{\max }$ and then decreases to zero effective stresses. The mean effective stress decreases continuously to zero, according to the formula:

$$
p^{\prime}=p_{0}^{\prime}-u
$$

where: $p_{0}^{\prime}=2=$ initial effective stress, $u=$ excess pore water pressure.

The equation that describes the effective stress path is the following:

$$
d \varepsilon_{v}=A d p^{\prime}+B \frac{\sqrt{3}}{2} \frac{d J_{2}}{\sqrt{J_{2}}}=0 .
$$

Using the constitutive functions $A$ and $B$ for initially contractive sand, we obtain the equation that describes the effective stress path for liquefaction: 


$$
\sqrt{J_{2}}=p^{\prime} \sqrt[4]{\frac{A_{v}^{u}\left(\sqrt{p_{0}^{\prime}}-\sqrt{p^{\prime}}\right)}{3 c_{1} \sqrt{p^{\prime}}}} .
$$

Fig. 16 shows experimental results and theoretical prediction for $A_{v}^{u}=2, c_{1}=5.8$ (equation (41)).

\section{Discussion and Conclusiona}

The most important results presented in this paper can be summarized as follows:

a) Original method of determining the pre-failure mechanical characteristics of granular soils under plain strain conditions is presented.

b) The model takes into account initial anisotropy and the initial state of sand (contractive or dilative), and applies to both fully drained and undrained conditions. It describes pre-failure deformations of sand and such phenomena as static liquefaction. Alternative definitions of loading and unloading are proposed.

c) $3 \mathrm{D}$ model has been calibrated using experimental data from triaxial experiments. Constitutive functions have been transformed, by the methods of tensor algebra, from triaxial to 3D conditions, including a special 2D case.

d) Predictions of the model for plain strain conditions and experimental results from investigations in TTA are presented. Agreement between experiments and the predictions of the model is quite good.

\section{References}

Alshibli K. A., Batiste S. N. and Sture S. (2003) Strain Localization is Sand: Plane Strain versus Triaxial Compression, Journal of Geotechnical and Geoenvironmental Engineering, ASCE, 129, 6, 483-494.

Chu J. and Wanatowski D. (2008) Instability Conditions of Loose Sand in Plane Strain, Journal of Geotechnical and Geoenvironmental Engineering, ASCE, 134, 1, 136-142.

Chu J. and Wanatowski (2009) Effect of Loading Mode on Strain Softening and Instability Behavior of Sand in Plane-Strain Tests, Journal of Geotechnical and Geoenvironmental Engineering, ASCE, 135, 1, 108-119.

Finno R. J., Harris W. W., Mooney M.A. and Viggiani G. (1997) Shear bands in plane strain compression of loose sand, Geotechnique, 47, 1, 149-165.

Jefferies M. and Been K. (2006) Soil Liquefaction. A Critical State Approach, Taylor and Francis, London and New York.

Lade P. V. and Yamamuro J. A., Editors (1999) Physics and Mechanics of Soil Liquefaction, Balkema, Rotterdam/Brookfield.

Madabhushi G., Knappett J. and Haigh S. (2010) Design of Pile Foundations in Liquefiable Soils, Imperial College Press, UK.

Menzies B. K. (1988) A Computer Controlled Hydraulic Triaxial Testing System, Advanced Triaxial Testing of Soil and Rock, ASTM, STP 977, Philadelphia, 82-94.

Mokni M. and Desrues J. (1998) Strain localization measurements in undrained plane-strain biaxial tests on Hostun RF sand, Mechanics of Cohesive-Frictional Materials, 4, 419-441. 
Oda M., Koishikawa I. and Higuchi T. (1978) Experimental study of anisotropic shear strength of sand by plane strain test, Soils and Foundations, 18, 1, 25-38.

Sawicki A. (2008) 3D and 2D Formulations of Incremental Stress-Strain Relations for Granular Soils, Archives for Hydro-Engineering and Environmental Mechanics, 55, 1-2, 45-53.

Sawicki A. (2011) Dilation and Stability of Sand in Triaxial Tests, Geotechnical Engineering Journal of the SEAGS and AGSSEA, 42, 4, 1-9.

Sawicki A. and Chybicki W. (2009) On accuracy of prediction of pre-failure deformations of granular soils, Computers and Geotechnics, 36, 6, 993-999.

Sawicki A. and Świdziński W. (2010a) Stress-strain relations for dry and saturated sands. Part I: Incremental model, Journal of Theoretical and Applied Mechanics, 48, 2, 309-328.

Sawicki A. and Świdziński W. (2010b) Stress-strain relations for dry and saturated sands. Part II: Predictions, Journal of Theoretical and Applied Mechanics, 48, 2, 329-343.

Sawicki A. and Świdziński W. (2010c) Modelling the pre-failure instabilities of sand, Computers and Geotechnics, 37, 6, 781-788.

Sumer M. (2014) Liquefaction around Marine Structures, World Scientific, New Jersey/London/Singapore.

Tatsuoka F., Sakamoto M., Kawamura T. and Fukushima S. (1986) Strenght and deformation characteristics of sand in plane strain compression at extremely low pressures, Soils and Foundations, 26, $1,65-84$.

Wanatowski D. (2007) Undrained instability of loose sand under plane-strain conditions and its engineering application, Foundations of Civil and Environmental Engineering, No. 10, 131-141.

Wanatowski D. and Chu (2007a) Drained behaviour of Changi sand in triaxial and plane-strain conditions, Geomechanics and Geoengineering: An International Journal, 2, 1, 29-39.

Wanatowski D. and Chu J. (2007b) $\mathrm{K}_{0}$ of sand measured by a plane-strain apparatus, Canadian Geotechnical Journal, 44, 1006-1012.

Wanatowski D. and Chu J. (2007c) Static liquefaction of sand in plane strain, Canadian Geotechnical Journal, 44, 299-313.

Wanatowski D. and Chu J. (2009) Instability behaviour of Changi sand in plane-strain tests, Proceedings of the $17^{\text {th }}$ International Conference on Soil Mechanics and Geotechnical Engineering, M. Hamza et al (Eds.), IOS Press, 89-92.

Wanatowski D., Chu J. and Loke W.L. (2010) Drained instability of sand in plane strain, Canadian Geotechnical Journal, 47, 4, 400-412. 